\title{
LES TABOUS DANS LE LEXIQUE OU QUAND LE CULTUREL CONDITIONNE LA VARIATION LEXICALE: LE CAS DE L'EUPHÉMISME ${ }^{1}$
}

TABOOS IN THE LEXICON OR WHEN THE CULTURAL CONDITIONS THE LEXICAL VARIATION: THE CASE OF THE EUPHEMISM

Lassâad Oueslati ${ }^{2}$

Resumo: Eeste artigo se propõe a mostrar a relação dialética entre léxico e cultura. Muitos fenômenos linguísticos confirmam a relação de entrelaçamento entre os dois. Isso pode observado no funcionamento da conotação, especialmente, no eufemismo. A cultura, por sua definição, permeia não apenas o modo de pensar de uma dada comunidade, mas também seu sistema linguístico. Nosso objetivo, neste artigo, é mostrar como a cultura pode condicionar a variação lexical. Para fazer isso, vamos examinar o eufemismo. Procuraremos mostrar em que medida essa figura de linguagem constitui uma das fontes de variação lexical e como ela permite contornar os tabus linguísticos.

Palavras-Chave: Competência lexical; Taxa cultural compartilhada; Idiossincrasia.

Article préparé en tant que produit du Projet Capes Cofecub 838/15.

Adresse e-mail de l'auteur: lassaadoueslati2020@gmail.com 
Abstract: We will try in this article to show the dialectical relation between lexicon and culture. Many linguistic phenomena confirm the entanglement relationship between the two. To be convinced of this, it suffices to see the functioning of the connotation, the tropes especially the understatement or the euphemism. Culture by its definition permeates not only the way of thinking of a given community but also its linguistic system. Our goal in this article is to show how culture can condition lexical variation. To do this, we will examine euphemism. We will try to show to what extent this figure constitutes one of the sources of lexical variation and how it makes it possible to circumvent linguistic taboos.

Keywords: Lexical competence; Shared cultural charge; Idiosyncrasy.

Résumé: Nous essayerons dans cet article de montrer la relation dialectique entre lexique et culture. De nombreux phénomènes linguistiques confirment la relation d'enchevêtrement entre les deux. Pour s'en convaincre, il suffit de voir le fonctionnement de la connotation, tropes la litote ou l'euphémisme. La culture de par sa définition imprègne non seulement le mode de pensée d'une communauté donnée mais aussi son système linguistique. Notre objectif, dans cet article, est de montrer comment la culture peut conditionner la variation lexicale. Nous examinerons, pour ce faire, l'euphémisme. Nous tenterons de montrer à quel point cette figure constitue une des sources de variation lexicale et comment elle permet de contourner les tabous linguistiques.

Mots-clés: Compétence lexicale; Charge culturelle partagée; Idiosyncrasie.

\section{INTRODUCTION}

L'impact culturel dans le lexique peut être abordé, non seulement en lexicographie, mais aussi d'autres disciplines telles que la philosophie, la sociologie, la sociolinguistique, la dialectologie, la didactique... Interrogeant le potentiel du culturel dans le vocabulaire dans une approche didactique, J.-C. Beacco (2000), affirme que "les mots ne traduisent pas directement, par leur sens enregistré en dictionnaire, les phénomènes culturels ${ }^{3 \prime \prime}$ (p.138). Il ajoute aussi qu'on peut repérer à travers les mots certains faits de société. En effet, les mots et, par conséquent la langue, constituent une représentation du monde, ne peut être que propre à chaque idiome. Le potentiel culturel ne peut donc être neutralisé. Aussi peut-on affirmer qu'il marque profondément la langue. Pour s'en convaincre, il suffit de tenir compte des expressions phraséologiques d'une langue qui comportent une dimension culturelle relative aux régions, aux sexes, aux générations utilisant la même langue. La traduction permet de dévoiler la charge culturelle véhiculée par les mots de la langue de départ et ceux de la langue d'arrivée.

L'euphémisme est un trope où le linguistique et le culturel se croisent. Il s'agit d'esquiver un mot fâcheux en employant un autre ménageant ainsi le locuteur. Or, un interdit linguistique est nécessairement motivé par un interdit

3 Beacco J.C. (2000), Les dimensions culturelles des enseignements de langue. Paris : Hachette. 
culturel, dans ce sens que l'on évite d'employer un vocabulaire chargé négativement dans la culture de l'interlocuteur. Rappelons-le protagoniste de la situation d'énonciation peut être de sexe différent, d'une autre génération, d'une autre région ou même pratiquant une autre langue. Ce qui revient à dire que le tabou linguistique peut caractériser le vocabulaire d'un sexe déterminé, d'une génération ou d'une localité.

Nous nous proposons dans cet article de nous pencher sur les expressions euphémistiques relatives aux deux sexes, aux régions et aux deux langues, en l'occurrence l'arabe et le français. Notre objectif est de mettre l'accent sur les manifestations de l'entremêlement entre langue et culture. Notre travail s'articulera ainsi autour des trois axes suivants : le premier axe se concentrera sur la délimitation du culturel dans la langue. Le deuxième abordera les différents mécanismes de l'expression linguistique du culturel. Le dernier axe examinera les divers cas de figure de l'euphémisme.

\section{RAPPORT ENTRE LANGUE ET CULTURE}

Dans son article intitulé «la compétence lexicale au service des représentations culturelles des apprenants en langues étrangères», J. Zajac ${ }^{4}$ cite André Martinet pour qui “Apprendre une langue étrangère, ce n'est pas mettre de nouvelles étiquettes sur des objets connus, mais s'habituer à analyser autrement ce qui fait l'objet de communication linguistique". Martinet pense ainsi que la langue n'est pas une simple série de signes en rupture avec le milieu culturel qui a forgé ces mêmes signes. Bien au contraire, le lexique et par conséquent la langue, sont loin d'être un code abstrait sans aucun lien avec la façon dont la communauté linguistique conçoit le monde. Cette idée nous conduit à penser que le rapport entre langue et culture est un objet de débat entre plusieurs disciplines. Par exemple, l'anthropologue français Claude LéviStrauss précise à ce propos que :

Le problème des rapports entre langage et culture est l'un des plus compliqués qui soient. On peut d'abord traiter le langage comme un produit de la culture; une langue en usage reflète la culture générale de la population. Mais dans un autre sens, le langage est une partie de la culture; il constitue un de ses éléments parmi

4 Zajac, J. (2009), "la compétence lexicale au service des représentations culturelles des apprenants en langues étrangères", dans Actes $d u$ congrès international, organisé par La Section de Linguistique et de Didactique des Langues et le Laboratoire de Didactique des Langues du Département de Langue et de Littérature françaises de l'Université de Thessalonique du 12 au Aristote 14 décembre 2008 sur le thème "2008 Année européenne du dialogue interculturel: Communiquer avec les Cultures-langues, pp. 588-596". 
d'autres. Mais ce n'est pas tout: on peut aussi traiter le langage comme condition de la culture (Lévi-Strauss 1958 in Van Der Sanden Piva 2001 : 21).

L'anthropologue montre clairement l'enchevêtrement entre langage et culture. La langue, pour lui, n'est pas seulement le miroir qui reflète le savoir partagé d'une population, mais elle est aussi le produit de la culture, ce qui instaure une relation dialectique entre les deux. Nous sommes, de ce fait, amené à nous poser la question sur la définition de cette dernière. Comment peut-on démêler ce qui est culturel de ce qui est langagier? Comment peut-on définir la culture?

Le Grand Robert définit la culture comme "un ensemble des aspects intellectuels d'une civilisation (...), des formes acquises des comportements, dans les sociétés humaines". En développant cette citation lexicographique, Mejri (2016) ramène la culture à "l'ensemble des pratiques, connaissances, normes et traditions transmises dans le cadre de la communauté ${ }^{\prime 5}$. Considérée comme un grand réceptacle des faits culturels, la langue, outil commun de communication entre les membres d'une même communauté, assure une fonction identitaire que chaque individu intériorise et assimile à son identité. Dans cette optique, l'appartenance géographique, civilisationnelle, religieuse, et par voie de conséquence culturelle, passe nécessairement par la langue. L'influence et l'interaction entre les deux s'avèrent tout à fait mutuelles. Ainsi se pose la question: comment se manifeste le culturel en langue?

En didactique des langues, on soulève souvent cette question du rapport entre langue et culture. Une conclusion s'impose: la langue est indissociable de la culture. D'ailleurs, R. Galisson, soulignant le jeu de symbiose qui gouverne le couple langue/culture, appelle les enseignants à "ne pas dissocier étude de la culture- étude de la langue, et vice-versa"6. À ce propos, il utilise la notion de “Charge Culturelle Partagée» qu'il définit en tant que «valeur ajoutée à leur signification ordinaire et pose que l'ensemble des mots à CCP connus de tous les natifs, circonscrit la lexiculture partagée"7. Pour cet auteur, la charge culturelle partagée témoigne de la relation entretenue entre le signe et ses utilisateurs. Pour illustrer cette idée, J. Zajac emprunte à R. Galisson (1991) l'exemple du signe "vache", lequel a, certes, le même signifié en France ou en Inde [femelle d'un taureau], mais la CCP diffère d'une langue à l'autre. En effet,

\footnotetext{
${ }^{5}$ Salah Mejri, 2016, "Les mots du jeu: entre idiomaticité et culture", in Les cahiers du dictionnaires: les mots de la méditerranée dans le dictionnaire, nº, p.30.

6 R. Galisson (1991, p. 119)

7 Idem, 120.
} 
en France, la vache est [exploitée parce qu'elle est nourricière] et en Inde, elle est [protégée parce que sacrée]. C'est ce qui conduit R. Galisson, (cité par J. Zajac, 2009, p. 591), à conclure $q^{\prime}$ « entre le mot français et son équivalent étranger, il y a couplage des Sés (signifiés) et découplage des CCP ${ }^{8}$.

Dans une autre optique, la culture est envisagée comme un facteur déterminant servant à inscrire une langue donnée dans l'universalité. C'est pour cela qu'on associe souvent l'universalité d'une langue à l'universalité d'une culture et vice versa. Robert Martin, menant une réflexion sur le concept d'universel dans la langue, soutient l'idée selon laquelle: "les grandes cultures, connues comme le patrimoine le plus précieux de l'humanité, sont indissociables de la langue qui les fonde. En conséquence, une langue peut être dite universelle dès lors qu'elle est la langue d'une culture universelle" ${ }^{\prime 9}$. Partant de l'idée que la conceptualisation des objets aussi bien que l'élaboration de la pensée sont deux opérations assurées par la fonction cognitive, R. Martin (2016) confirme le rapport indissociable entre la langue et la culture, sachant que cette dernière est assimilée au réel. Il conclut ainsi qu' «une langue est un système qui structure le réel, qui organise le pensable, qui conceptualise les données du monde» ${ }^{10}$. La «fonction expressive » de la langue vient, selon Martin, appuyer la dimension culturelle. L'aboutissement de cette fonction est à la fois « esthétique et littéraire», ce qui favorise l'universalité de la langue. Autrement dit, bien que la communication soit une fonction importante définissant la langue, la dimension créative de la langue, qui apparait à travers la production esthétique et littéraire, favorise plus l'universalité de cette langue. D'ailleurs, pour découvrir les richesses culturelles d'une langue, il suffit d'accéder à sa production littéraire, scientifique et philosophique. Mejri (2016) affirme dans ce sens que «c'est dans l'enracinement culturel et linguistique que se nourrit l'universalité».

Nous avons tenté, dans cette partie, de mettre en lumière le rapport entre langue et culture. Il s'avère que, en dépit de la diversité des approches et des angles d'attaque (didactique, anthropologique, linguistique ou logicosémantique), il y a une convergence entre les différents points de vue pour affirmer l'indissociabilité entre les deux. Étudier une langue n'est pas réductible à l'étude de sa syntaxe, de sa sémantique, de sa morphologie, de sa pragmatique, etc. C'est aussi étudier l'aspect culturel sous-jacent qui se

${ }^{8}$ Galisson, p. 120

${ }^{9}$ R. Martin, 2016, p. 81

${ }^{10}$ Idem, 80 
manifeste à tous les niveaux. Nous nous proposons dans ce qui suit de voir les principales manifestations linguistiques de l'aspect culturel.

\section{LES MANIFESTATIONS DU CULTUREL DANS LA LANGUE}

Dans son ouvrage Linguistique cognitive, comprendre comment fonctionne le langage (deuxième édition, Duculot, 2010), N. Delbecque affirme que "la signification, c'est-à-dire la conceptualisation linguistique, peut varier fortement d'une langue et d'une culture à l'autre"11. Pour elle, le linguistique et le culturel s'entremêlent à tous les niveaux: lexicologique, morphologique, syntaxique et même phonologique. Face à cet enchevêtrement entre les deux, on retient parmi les approches linguistiques deux positions extrêmes. La première "défend l'idée de la relativité linguistique"12. Il s'agit d'une approche pour laquelle "le rapport que l'on entretient avec le monde serait modulé par la langue et la culture"13. La deuxième, qui est aux antipodes de la première, est celle de l'universalisme. Cette approche "postule que, l'esprit humain étant inné, la pensée humaine est foncièrement la même, et ce, quelles que soient la langue et la culture"14. Pour cette approche, «le fonds commun de l'unité "psychique de l'humanité" sert de source dans laquelle puisent les langues et cultures du monde. Poussée à l'extrême, cette approche ramène toutes les langues, aussi diverses soient-elles, à une conceptualisation linguistique identique.

Trouvant que ces deux approches sont extrêmes, irréconciliables, la démarche cognitiviste que défend N. Delbecque consiste à trouver une voie intermédiaire dans la mesure où ces deux positions, malgré leur excès, couvrent une certaine vérité. La solution médiane consiste à dire que «si la plupart des catégories conceptuelles paraissent effectivement inscrites dans une langue et une culture bien spécifiques, il y a néanmoins un petit nombre de catégories conceptuelles qui se retrouvent dans toutes les langues et dans toutes les

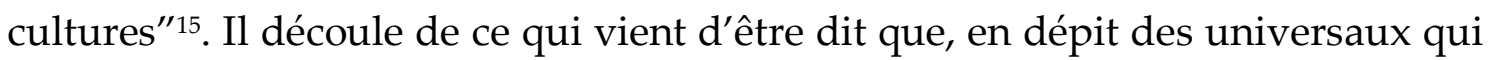
caractérisent le langage humain, il y a néanmoins une spécificité culturelle imprégnant toute langue, laquelle spécificité apparait à travers la relativité

11 Delbecque N., 2010: 163

12 Idem

13 Ibidem

14 Ibidem

15 Idem, 164 
linguistique et culturelle, le lexique culturellement marqué et les grammaires culturellement spécifiques.

Ce qui est de la relativité linguistique, il s'agit de défendre l'idiosyncrasie, principe qui consiste à reconnatre à chaque langue des spécificités qu'on ne trouve nulle part ailleurs. Ce principe reconnait l'existence $d$ 'interaction et d'influence mutuelle entre langue et culture. Autant de langues autant de cultures. N. Delbecque dresse une liste de philosophes, poètes, philologues, etc. qui, depuis le XVII ${ }^{e}$ siècle, reconnaissent l'impact de la culture sur la langue. Elle cite à ce propos le philosophe anglais John Locke qui, depuis 1690, "fit valoir que dans toutes les langues il y a "bon nombre de mots...auxquels on ne saurait trouver d'équivalents dans aucune autre [langue]"16. Les mots sont, pour lui, un vrai miroir reflétant les coutumes et les modes de vie. Dans le même ordre d'idées, elle cite le poète allemand Johann Gottfried et le philologue Wilhelm von Humboldt (1767-1835) qui soutiennent l'interdépendance entre la langue et la culture. Par exemple, dans son ouvrage De l'hétérogénéité des langues et de leur influence sur le développement mental de l'humanité (1827-1829), Wilhelm von Humboldt «voit les langues comme des prismes ou des grilles recouvrant la réalité extralinguistique, de sorte que chaque langue reflète sa propre vision du monde ${ }^{17}$ ». Cette idée fut à l'origine de la fondation de l'anthropologie culturelle et linguistique, nouvelle discipline instaurée par l'Américain Franz Boas. Partant de l'étude des langues et cultures indiennes dans le Nouveau Monde, cet anthropologue et ses disciples retiennent l'extrême différence des catégories conceptuelles entre les cultures et les langues indiennes et européennes. Cette différence se manifeste notamment dans le lexique; ce qui amène Edward Sapir (1949, p. 27) à conclure que "distinctions qui nous paraissent indispensables sont parfois entièrement ignorées dans les langues qui, d'une part, reflètent une culture totalement différente, mais qui, d'autre part, insistent sur des distinctions que nous trouvons tout à fait inintelligibles"18.

L'enchevêtrement entre le linguistique et le culturel et leur influence mutuelle ne concerne pas exclusivement le lexique. Bien plus, ils peuvent influencer aussi les conceptualisations grammaticales. Benjamain Lee Worf (1887-1941), disciple de Sapir, met en place les théories de la relativité linguistique. Ce chimiste, qui s'intéresse à l'anthropologie, parvient à montrer que toute langue dispose des structures habituelles qu'il appelle "patrons

16 Ibidem

17 Ibidem

18 Cité par Delbecque, 2010: 165 
habituels", structures qui exercent nécessairement un pouvoir sur la façon de voir et de penser des locuteurs. Pour preuve, il suffit de voir les paraphrases: "Quand on paraphrase, il y a toujours un prix à payer: il nous faut recourir à des tournures plus longues, plus complexes, plus lourdes que celles qui se conforment aux structures grammaticales ordinaires" ${ }^{\prime 19}$. Et Delbecque d'ajouter que, quelle que soit la langue maternelle, ses structures habituelles influencent imperceptiblement les façons de voir et de penser des pratiquants de cette langue. En effet, le découpage du réel n'est pas le même d'une culture à l'autre. Pour s'en convaincre, il suffit de voir le phénomène de la paraphrase d'un mot dans une autre langue. Comme la plupart des mots n'ont pas d'équivalents exacts dans chaque langue, le recours à une paraphrase équivalente devient nécessaire. N. Delbecque cite à titre d'exemple le mot français main, lequel mot ne couvre pas le même champ notionnel du mot russe ruka. Ce dernier désigne à la fois le bras entier et la main. Elle cite également le cas du verbe casser. Son équivalent en malais change "selon que la cassure est complète ou partielle" 20. On obtient ainsi, selon le type de cassure, soit putus soit patach.

Au-delà de leur sens culturellement marqué, certains mots sont, euxmêmes, culturellement appropriés à une langue donnée. Leur spécificité va de pair avec la spécificité d'un produit, d'un objet, bref d'un référent extralinguistique quelconque. Ainsi, une langue, ne disposant pas du référent exact que désigne un mot simple dans une langue étrangère, a recours à une paraphrase au cas où elle n'emprunte pas ce mot tel quel. Par exemple, le plat polonais bigos a pour équivalent en français le nom composé "ragoût aux choux", barszcz son équivalent est "soupe aux betteraves" et powid-a est traduit par "confiture aux prunes". De même, le mot dattes est le mot générique auquel correspondent quelques centaines de variétés de dattes au sud tunisien ${ }^{21}$, renvoyant chacune à un type précis de dattes. Delbecque ramène ce phénomène à la spécificité culturelle du domaine décrit. Elle précise à ce propos que "d'une langue à l'autre, l'inventaire des mots culturellement spécifiques sera plus ou moins grand en fonction de l'importance accordée au domaine auquel ils se rapportent" ${ }^{\prime 22}$. Elle ajoute: "le phénomène de l'élaboration lexicale, qui consiste à privilégier le développement du lexique propre à certains domaines plutôt qu'à d'autres est révélateur des conditions de vie du pays ou de la région. L'abondance, la précision et la variété du vocabulaire sont directement

19 Idem, 167

20 Idem, 171

21 Les enquêtes de l'Atlas linguistique de Tunisie le confirment.

22 Delbecque, 2010, p. 173 
proportionnelles à l'impact du domaine en question sur le mode de vie" ${ }^{\prime 23}$. Ainsi le lexique d'une langue augmente ou se réduit proportionnellement à l'expérience que l'on vit selon que celle-ci est relative au milieu social, à l'activité économique, au sexe, à l'appartenance sociale, etc. Pour avoir une idée sur le lien étroit entre la langue et les facteurs extralinguistiques que nous venons d'énumérer, il suffit de voir les technolectes ${ }^{24}$.

Outre l'impact qu'elle peut avoir sur le lexique d'une langue, la culture peut également influencer profondément la grammaire qui régit cette même langue. De nombreuses catégories syntaxiques marquent pour ainsi dire la différence d'une langue par rapport aux autres langues. Pour illustrer cette idée, prenons à titre d'exemple l'expression du nombre, celle de l'intensité et celle du DouSa. En ce qui concerne la première, celle du nombre, l'arabe, par exemple, contrairement à d'autres langues, exprime le duel. En effet, l'arabe réserve un tiroir de conjugaison réservé à la catégorie syntaxique du duel, en faisant la distinction entre le duel du présent أنتما) مثنى الحاضر) (Pantuma) et le duel de l'absent هما مثنى الغائب (huma). Cependant, dans d'autres langues comme le français, la catégorie du pluriel couvre tous les nombres supérieurs à un, le duel étant assimilé au pluriel, par opposition au singulier. L'arabe distingue aussi le duel féminin du duel masculin: هما ذهبنا/ هما ذهبا [huma Sahaba]/ [humE Sahabata:].

Quant à l'intensité, elle se caractérise par sa fonction expressive. Cette expressivité semble correspondre en italien à l'expressivité générale de cette culture. En effet, les Italiens ont recours à la réduplication pour exprimer une charge émotionnelle intensive. Les constructions bella bella (" belle, belle"), adagio adagio ("lentement, lentement"), subito subito ("immédiatement, imméditament") n'admettent pas, selon N. Delbecque ${ }^{25}$, la paraphrase par l'adverbe de degré très. Elle en conclut que « la signification de la réduplication italienne va bien plus loin que celle de três". Elle joute que la traduction bella bella par "très belle" "en affadit le sens"26.

Dans le même ordre, l'expression de l'intensité en arabe tunisien se fait

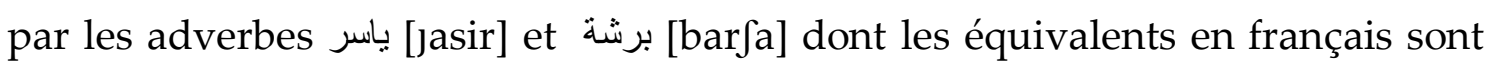
"trop" et "beaucoup". Outre ces deux adverbes, l'intensité peut être exprimée par l'intonation. Par exemple, pour dire qu'un endroit est "trop loin" ou qu'un niveau est "trop profond", on exprime l'intensité en parler tunisien en

23 Idem

24 Oueslati L (1999).

25 Delbecque N., 178

26 Idem, 179 
allongeant une voyelle bien déterminée. Nous prononcerons consécutivement

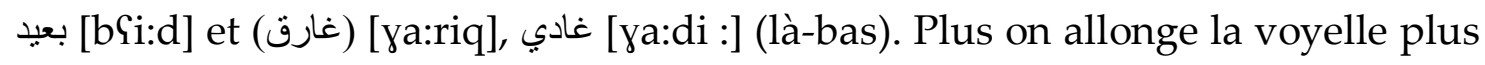
on exprime l'intensité. Mais l'expression de l'intensité au moyen de l'intonation n'est pas équivalente aux adverbes d'intensité. La preuve, c'est qu'on peut intensifier davantage les adverbes de degré en arabe tunisien par l'intonation, ce qui fait que ces adverbes sont sous la portée de l'intonation alors que l'inverse n'est pas vrai. Ce phénomène de l'intonation serait au parler tunisien ce qu'est la réduplication à l'italien.

Le Duৎa relève de la phraséologie. Bien qu'il soit traduit en français par invocation, ce genre de discours est propre à la culture arabo-musulmane, raison pour laquelle on le trouve aussi bien dans l'arabe littéraire, où l'influence du Coran n'est plus à démontrer, queles parlers arabes. Avoir recours à ce genre de est un indice d'appartenance religieuse, en l'occurrence l'Islam. À l'intérieur de ce genre discursif et phraséologique, qu'est le $D u \uparrow a$, nous pouvons distinguer de nombreuses sous-classes. Il existe en tunisien par exemple un DuSa pour le pèlerinage (إن شاء الله في عودة) [?infa?a alah fi ؟awda] (trad. J'espère que vous aurez l'occasion d'y retourner). D'autres formes sont destinées aux parents après l'accouchement de la mère: إن شاء الله من الذرية الصالحة [?infa?a alah mina orija] (trad. J'espère qu'il/elle sera de la bonne semence). Outre l'appartenance religieuse, certains $D u S a$ peuvent indiquer l'appartenance du locuteur à une confession religieuse, telle que celle des salafistes. D'autres types peuvent indiquer le sexe du locuteur comme certains DuSa spécifiques aux parlers des femmes. Pour avoir une idée plus précise sur ce genre de phraséologie, nous renvoyons aux travaux de Néji Kouki ${ }^{27}$ et Béchir Ouerhani ${ }^{28}$.

Nous avons tenté dans ce qui précède de montrer l'omniprésence du culturel dans le linguistique. Notre constat principal est que les différents niveaux de la langue marquent une certaine interférence entre l'individuel et le collectif, le fantaisiste et le consensuel, bref entre le linguistique et le culturel. La culture imprègne ainsi la langue, de l'intonation jusqu'au texte. Même la grammaire, qui est supposée être neutre, n'échappe pas à l'impact de la culture.

27 Néji Kouki, «Les énoncés du Duৎa:?, l'invocation : traitement de la variation régionale dans un continuum entre l'arabe littéral et le dialecte tunisien ", dans Les cahiers du dictionnaire: les mots de la Méditerranée dans le dictionnaire, sous la direction de Giovanni Dotoli et Salah Mejri, Classiques Garnier, pp. 347-367

28 Béchir Ouerhani, «Les énoncés du DuYa:?, Données empiriques et description linguistique», dans Les cahiers du dictionnaire : les mots de la Méditerranée dans le dictionnaire, sous la direction de Giovanni Dotoli et Salah Mejri, Classiques Garnier, pp. 369-383 
Nous nous proposons dans la partie suivante d'aborder le degré d'influence que subit une figure telle que l'euphémisme par le phénomène culturel.

\section{L'EUPHÉMISME: UN CAS D'ENCHEVÊTREMENT ENTRE LANGUE ET CULTURE}

La question centrale à laquelle nous essayons de répondre dans cet article est de savoir quel lien on établit entre l'euphémisme et la culture. Cette question nous conduit à rappeler la définition de cette figure du discours. L'euphémisme est défini comme "essentiellement un fait social, étant donné qu'il trouve son origine dans l'action, la pression exercée par la collectivité sur

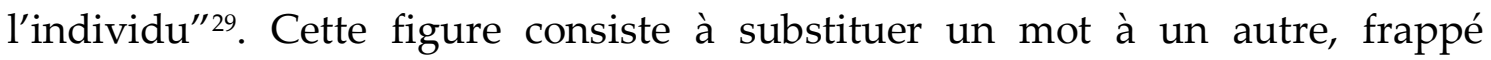
d'interdit. Il s'ensuit que l'euphémisme est variable dans la mesure où les sociétés aussi bien que les époques varient, et ce qui est interdit dans une société ou dans une époque peut ne pas l'être dans une autre ou peut ne plus l'être, tellement le phénomène linguistique est aléatoire et variable tout comme l'échelle des valeurs. Ainsi l'individu se trouve-t-il conditionné par la culture du groupe. C'est à ce propos que John Orr (1953) soutient que "c'est le groupe, avec toutes les croyances, tous les préjugés qui le caractérisent et qui soulignent son homogénéité, qui frappe d'interdit tel mot ou telle expression et oblige l'individu soit au silence absolu - c'est l'euphémisme 'zéro', l'euphémisme 'absence', (...) soit à 'tourner autrement'”'30.

Nous en concluons que l'euphémisme est un phénomène à la fois linguistique et social. En effet, il s'agit d'une figure d'atténuation cherchant à émousser, à édulcorer ou à atténuer un sens choquant. Cette figure a recours à de nombreux procédés dont la litote, la paraphrase ou "d'autres formes de substitution lexicale comme l'antiphrase ou la métaphore" ${ }^{\prime 31}$. Nous en déduisons également la relativité de l'interdiction de telle ou telle expression selon le cadre spatio-temporel. Par exemple, J. Orr (1953) cite à ce propos l'exemple du verbe baiser, avait un emploi euphémistique qui remonte au $X^{e}$ e siècle et même plus. Ce même verbe a perdu sa valeur euphémistique au XVIe siècle et est devenu, de par sa brutalité, un mot cru, considéré comme un tabou.

29 Orr John, «Le rôle destructeur de l'euphémie » In: Cahiers de l'Association internationale des études françaises, 1953, n³-5. pp. 167-175

30 Idem, p.157

31 C. Kerbrat-Orecchioni (1994), citée par Anna Jaubert, 2008/4 : 107), «Dire et plus ou moins dire. Analyse pragmatique de l'euphémisme et de la litote», Langue française ( $\left.{ }^{\circ} 160\right)$, p. 105116.) 
Pour se convaincre de la relativité de l'interdit qui pèse sur une expression ou une autre, il suffit de voir le parler tunisien. En effet, la description de ce dialectal dans le cadre de l'Atlas Linguistique de Tunisie nous permet de nous rendre compte que le tabou, et par conséquent l'euphémisme, varie d'une époque à l'autre, d'un sexe à l'autre et d'une société à l'autre. Les données lexicales recueillies lors des enquêtes du terrain effectuées par l'équipe de l'Atlas Linguistique de Tunisie nous permettent de dégager trois cas de figure: le premier concerne des mots tabous pour quelques régions; le second cas de figure, des expressions interdites par le code social dans toute la Tunisie; le dernier cas de figure, la relativité de l'euphémisme par rapport à une autre langue, en l'occurrence le français. Le point commun entre ces trois cas de figure est l'interférence entre le culturel et le linguistique. Les comparer, tout dépend du niveau de l'interdit qui frappe le mot: dans le premier, il s'agit d'un tabou à une échelle locale, communautaire, dans le deuxième cas, c'est à l'échelle nationale que le mot est connoté péjorativement et le dernier cas concerne des tabous à l'échelle interlinguale.

S'agissant du premier cas, certains mots sont frappés d'interdit dans certaines régions en Tunisie et sont neutres dans d'autres. Nous citons, à titre

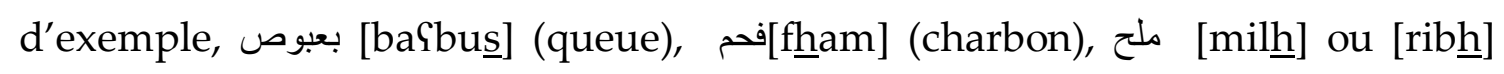
(sel). Ces mots, bien qu'ils soient dans certaines régions tunisiennes neutres, sont dans d'autres régions connotés négativement. Par exemple, le premier mot, [baSbus] (queue), est frappé d'interdit notamment dans les régions du sud et celle du centre où il connote le membre viril. Son emploi dans les régions du nord ou celles du nord ouest ne comporte pas la charge sémantique négative qu'il a dans les autres régions. On lui substitue donc le mot neutre de l'arabe

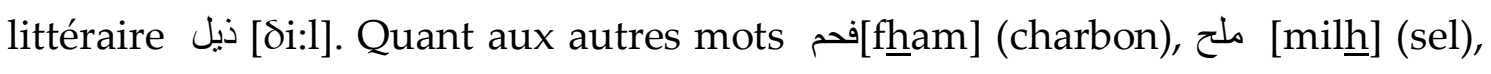
ils ont, dans certaines régions et chez une génération d'une tranche d'âge supérieure à la soixantaine, une charge sémantique très négative. Le premier,

فحم [fham] (charbon), a une connotation négative provenant du noir, couleur prototypique du deuil ou de l'esclavage. On lui substitue بياض [bja:ㅇ] (blancheur). Le second, ملح [milh]], est associé à la perte puisqu'il se définit par sa dissolution en contact avec l'humidité. C'est pour cette raison qu'on le remplace dans certaines régions par [ribh]], (gain), c'est-à-dire l'antonyme du sens connoté.

Toujours dans le premier cas de figure où le tabou concerne une communauté bien déterminée, nous pouvons citer des tabous propres à un sexe particulier. Par exemple, la manière dont les femmes désignent la menstruation 
selon la région ou selon les tranches d'âge. Substitue au mot règles, mot جاوني : considéré comme tabou, d'autres mots paraphrasant le premier. On dit [3a:wu:ni] (trad. Elles me sont arrivées) (trad. Je les ai eues), مريضة [mri:ㅁa] (trad. malade), مستخة [masxa] (trad. sale). Pour les jeunes filles qui viennent d'atteindre la puberté et d'avoir leurs premières règles, on désigne cet événement par une phrase euphémistique بشرت [baكrit] (trad. Elle a annoncé la فلانة تغسل و تنشر bonne nouvelle) ou on peut avoir recours à une phrase figée [foulana taxsil w tãfur] (trad. telle lave et étend son linge). Il découle de ces exemples que l'euphémisme est à l'origine d'une certaine variation lexicale. En effet, le même mot peut être investi d'une nouvelle charge sémantique permettant d'atténuer le sens premier d'un mot substitué considéré comme choquant. C'est le cas, par exemple, du mot ظروف [ôuru:f] qui signifie littéralement "conditions" ou "circonstances". Ce mot, prononcé par une femme égyptienne dans la phrase figée عندي ظروف [؟ãdi ôuru:f], signifie que la femme en question a ses règles.

En ce qui concerne le tabou qui frappe certains mots à l'échelle nationale, on le trouve dans de nombreuses expressions et dans divers mots. Il témoigne d'une certaine unité culturelle dans la mesure où il s'agit du même code social et moral. Aussi les tabous relèvent-ils du registre religieux. Pour mieux comprendre cette idée, nous prenons l'exemple du vin, de l'organe génital et des relations sexuelles en dehors du cadre légitime. En ce qui concerne le vin, il est désigné par le mot générique شراب[كra:b]. Ce dernier signifie littéralement "boisson". Ce qui signifie qu'il a un emploi euphémistique du mot خمر [xamr]. Mais le mot [ $\left.\int \mathrm{ra}: b\right]$, tellement spécialisé dans la désignation du vin, produit dont la consommation est interdite par l'Islam, est lui même frappé d'interdit. On lui substitue ainsi par métonymie le mot معصية [muYsja], [trad. péché]. Son emploi métonymique euphémistique apparat dans l'expression نشرب في المعصية [nufrub filmu@sja] (trad. Je bois le péché).

De même, l'adultère ou toute relation en dehors du cadre du mariage sont considérées comme des péchés. Aussi ces actes sont-ils désignés autrement de façon euphémistique. Par exemple, la majorité des Tunisiennes et Tunisiens, pour désigner une relation sexuelle illégale disent عندي/ ها كا (trad. J'ai/ elle a une situation). Les journalistes des pages de faits divers parlent du viol ou des

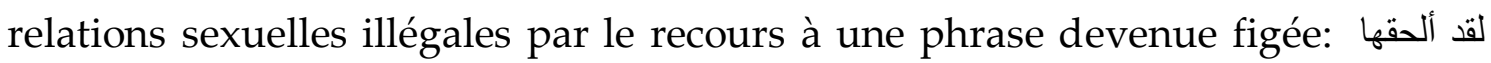
بصفوف النساء المنزوجات [laqad Palhaqaha bisufufi Pnisa? al mutazwi3?a:t] (trad. Il la fait rejoindre les rangs des femmes mariées). Dans le même ordre d'idées, les appareils génitaux masculin et féminin sont considérés comme tabous. Ils sont 
désignés différemment. L'expression euphémistique la plus générique est le pronom possessif qui désigne les deux appareils, selon le référent. On dit en effet مناعي/ متاعو / متاعها [mta:Si] [mta:Yu] [mta:ha] (trad. Le mien). Rappelons à ce propos que dans la majorité des régions tunisiennes, on désigne l'appareil génital de l'homme par محاثم [mạa:jim] (trad. honte) et l'appareil génital de la femme par سُترة [sutra] (trad. sa discrétion). De même, en Tunisie, comme la société est conservatrice, certains mots sont tabous dans toutes les régions, étant donné qu'on se réfère au même code moral. C'est le cas du mot قحبة [qahba] (trad. pute). Étant donné la charge sémantique très négative de ce mot, il est remplacé systématiquement par des paraphrases telles que مرا ما تصلحش [mra: ma:taslihhf] (trad. Une femme qui n'est pas valide), c'est-à-dire une femme de mœurs légères.

Quant au dernier cas de figure, il relève de la différence entre les langues, une autre preuve qui montre la relation intime entre le culturel et le linguistique. En effet, ce qui est tabou dans une culture ne l'est pas nécessairement dans une autre et vice versa. Par exemple, le mot foutre avait, selon J. Orr ${ }^{32}$, un substitut euphémistique au XVI ${ }^{\mathrm{e}}$ siècle, à savoir outre. Mais avec le temps, ce verbe, employé en langage familier, a subi un dépérissement fonctionnel dans ce sens qu'il ne renvoie plus au registre sexuel. Ainsi foutre quelque chose parterre, se foutre de qqn, se foutre dedans, etc. ne sont plus choquants en français contemporain. Cependant, l'équivalent en tunisien garde sa valeur connotative sexuelle choquante pour les usagers de ce parler. On a de ce fait recours à "l'euphémisme zero", c'est-à-dire au silence. Il s'agit là d'une preuve tangible de l'impact culturel sur la langue. De plus, comme la culture évolue et les valeurs de sociétés, les langues, de par leur fonction communicative, reflètent fidèlement cette évolution en les mots aux différents savoirs qu'ils véhiculent.

\section{CONCLUSION}

En abordant la question de l'impact du culturel sur la langue, nous avons de façon préalable la conviction que la langue n'est pas réductible à un code de communication dépourvu de toute couleur ou de toute appartenance sociale ou culturelle. Nous avons même l'intime conviction que la langue, outre sa fonction communicative, est un miroir reflétant fidèlement la façon dont l'usager de cette langue conçoit le monde. En effet, le même référent n'est pas

32 Op. cit. p.169. 
conçu de la même façon dans les différentes communautés utilisant la même langue mais adoptant des valeurs morales différentes. L'euphémisme s'avère une piste de recherche prometteuse puisqu'il s'agit d'utiliser la langue pour émousser un sens choquant dans un code culturel donné.

Il nous reste à souligner enfin l'importance de l'euphémisme, ce phénomène complexe qui se trouve à la croisée des opérations relatives à la circulation du sens telles que la synonymie, l'expression de l'altérité, etc. C'est un phénomène qui nous conduit à la lisière de ce qui ne doit pas être dit, où se mêlent le linguistique, le culturel, le psychologique, le sociologique, etc. Il serait intéressant d'examiner davantage l'euphémisme pour délimiter ce qui sépare le linguistique du culturel. Un tel travail pourrait déboucher sur des mécanismes universaux permettant d'occulter ce qui est fâcheux, ce qui est une façon d'isoler le culturel, et donc le relatif du général ou de l'universel.

\section{RÉFÉRENCES}

BEACCO, J.C. Les dimensions culturelles des enseignements de langue. Paris: Hachette, 2000.

DELBECQUE, N. Linguistique cognitive, Comprendre comment fonctionne le langage, Nouvelle édition augmentée, avec exercices et solutions. Bruxelles: De boeck Duculot, 2010.

GALISSON, R. De la langue à la culture par les mots. Paris: Clé International, 1991.

KERBRAT-ORECCHIONI, C. Rhétorique et pragmatique: les figures revisitées, Langue française, $\mathrm{n}^{\circ} 101$, p. 57-71, 1994.

KOUKI, N. Les énoncés du Duৎa :?, l'invocation: traitement de la variation régionale dans un continuum entre l'arabe littéral et le dialecte tunisien. In: Dotoli, Giovanni; Mejri, Salah. Les cahiers du dictionnaire: les mots de la Méditerranée dans le dictionnaire. Paris: Classiques Garnier, 2016, p. 347-367.

MARTIN, R. Linguistique de l'universel, Réflexions sur les universaux du langage, les concepts universels, la notion de langue universelle. Paris: Académie des Inscriptions et Belles-Lettres, 2016.

MEJRI, S. Les mots du jeu: entre idiomaticité et culture. In: Dotoli, Giovanni; Mejri, Salah. Les cahiers du dictionnaires: les mots de la méditerranée dans le dictionnaire, Paris: Classiques Garnier, 2016, p. 29-46.

ORR, J. Le rôle destructeur de l'euphémie. Cahiers de l'Association internationale des études françaises, Paris, n³-5, 1953, p. 167-175.

OUERHANI, B. Les énoncés du DuYa:?, Données empiriques et description linguistique. In: DOTOLI, Giovanni; MEJRI, Salah. Les cahiers du dictionnaires: les mots de la méditerranée dans le dictionnaire. Paris: Classiques Garnier, 2016, p. 369-383. 
OUESLATI, L. De l'identique au différent: le parcours d'un euphémisme. In: CLASS, A.; AWAISS, Henri. Actualité Scientifique, L'éloge de la différence: la voix de l'autre. Liban: VIèmes journées scientifique du Réseau thématique de l'AUF, Lexicologie, Terminologie, Traduction, 1999, p. 191-197

OUESLATI, L. De l'identique au différent: le parcours d'un euphémisme. In: BACCOUCHE, Taïeb; MEJRI, Salah. Langage et Altérité: l'expérience de l'Atlas Linguistique de Tunisie. Tunisie: C.R.E.S, 2001, p.99-108

ZAJAC, J. La compétence lexicale au service des représentations culturelles des apprenants en langues étrangères. In: Actes du congrès international, 2008, Section de Linguistique et de Didactique des Langues et le Laboratoire de Didactique des Langues du Département de Langue et de Littérature françaises de l’Université de Thessalonique du 12 au Aristote 14 décembre 2008 sur le thème Année européenne du dialogue interculturel: Communiquer avec les Cultures- langues. Atenas: 2009, p. 588-596.

Nota do editor:

Artigo submetido para avaliação em: 06 de dezembro de 2018.

Aprovado em sistema duplo cego em: 18 de dezembro de 2018. 